\title{
Designing a Markov Model for the Analysis of 2-tier Cognitive Radio Network
}

\author{
Tamal Chakraborty \\ Department of Electronics and Telecommunication \\ Engineering \\ Jadavpur University \\ Kolkata, India
}

\author{
Iti Saha Misra \\ Department of Electronics and Telecommunication \\ Engineering \\ Jadavpur University \\ Kolkata, India
}

\begin{abstract}
Cognitive Radio Network (CRN) aims to reduce spectrum congestion by allowing secondary users to utilize idle spectrum bands in the absence of primary users. However, the overall user capacity and hence, the system throughput is bounded by the total number of available idle channels in the system. This paper aims to solve the problem of limited user capacity in basic CRN by proposing a 2-tier CRN that allows another tier (or layer) of secondary users to transmit, in addition to the already existing set of primary and secondary users in the system. Markov Models are designed step-wise to map the interaction between primary and secondary users in both tiers by including suitable traffic distribution models and system parameters. Spectrum handoff is also incorporated in the developed Markov Models. Performance analysis is carried out in terms of SU transmission, dropping, blocking and handoff probabilities along with mathematical formulation of the overall SU throughput in 2-tier CRN. It confirms better spectrum utilization in spectrum handoff enabled 2-tier CRN over basic CRN with enhancement in quality of service for secondary users in terms of reduced dropping and blocking probabilities.
\end{abstract}

Keywords-Cognitive Radio Network; 2-tier; Voice over IP; Markov Model; Spectrum Handoff

\section{INTRODUCTION}

Wireless communication has witnessed increased popularity owing to rapid development of mobile and portable applications that have enabled users to communicate "anytime anywhere". This has led to formulation of admission control and network management policies to deal with the problems of scalability, fairness, synchronization and security, that arise with increased subscribers in wireless domain. Recent studies $[1,2]$ have clearly demonstrated that while spectrum congestion hinders further growth in wireless communication, there are plenty of idle spectrum regions that are left unutilized. Cognitive Radio Network (CRN) [3-5] aims to create a common spectrum pool by including all such unused spectrum bands and allocate them to applications based on their requirements. It deploys opportunistic mode of communication where secondary or unlicensed users (SUs) transmit in the frequency slots when the corresponding primary or licensed users (PUs) are absent. However, practical implementation of CRN must address the issues of spectrum analysis, management and mobility, along with architectural specifications [6].

Extensive research work is being carried out to achieve higher spectrum utilization in CRN through formulation of appropriate Medium Access Control (MAC) protocols [7, 8], handoff schemes [9], timing parameters [10, 11], etc. However, the system capacity in all these works has a maximum upper bound as derived in [12]. This paper introduces the concept of "2-tier CRN" that increases the capacity of CRN by admitting more number of SUs in the network. The SUs are categorized into two tiers. The SUs in the first tier are Voice over IP (VoIP) [13] users that transmit in the secondary transmission interval when the PUs are sensed idle. The second tier of SUs performs data transmission during the silence periods of VoIP SUs in the first tier. To the best of our knowledge, no such work has been reported on this issue so far as primarily, research has been carried out in CRN comprising of only one tier of SUs.

Markov Model serves as an effective tool to design CRN and has been implemented widely in recent works $[14,15]$. The primary advantage of developing CRN with Markov Model is that it incorporates user-defined traffic distribution for PUs and SUs, along with customized network conditions and thereby, facilitates study of the complex interaction between $\mathrm{PU}$ and SU in CRN. Accordingly, the objective of this paper is to design Markov Models for basic and 2-tier CRN and analyze the increase in system capacity of 2-tier CRN over basic CRN with respect to SU dropping, blocking, handoff and transmission probabilities. A mathematical framework is also established that calculates the SU throughput for a complete spectrum handoff enabled 2-tier CRN.

The paper is organized as follows. The principle for 2-tier CRN is discussed in Section II. Markov Models for the first and second tier of 2-tier CRN are described in Section III along with spectrum handoff in these networks. Section IV provides mathematical model to calculate SU throughput in 2-tier CRN followed by performance analysis in Section V.

\section{Principle of 2-Tier Cognitive Radio Network}

The proposed 2-tier CRN consists of one tier of PUs and two tiers of SUs. PUs are allotted designated channels for transmission. When PUs are not transmitting, the idle channels are utilized by SUs. SUs are categorized into VoIP SUs and DATA SUs. VoIP SUs demand higher Quality of Service (QoS) and hence, have priority over the DATA SUs in accessing idle channels. Therefore, channels are utilized by SUs in the following manner.

- Whenever a licensed channel is sensed idle, VoIP SU occupies the channel and starts transmission. As VoIP 
transmission occurs in talkspurts [16], there are idle periods of inactivity that are detected by codecs. Silence suppression [16] is performed, thereby making channel accessible to other users. At the onset of another talkspurt, the channel is reclaimed back by the VoIP SU for transmission. VoIP SU, therefore, occupies the first tier of 2-tier CRN and is denoted by SUtier1.

- DATA SU utilizes the channel during "off" period of SUtier1 and continues transmission until the channel is either reclaimed back by VoIP SU or is sensed busy at the end of secondary transmission time slot. These SUs implement queuing models to reduce packet loss when connection is terminated. DATA SU, thus, constitutes the second tier of 2-tier CRN and is denoted by SUtier2. Fig. 1.

The principle of 2-tier CRN is depicted in a flowchart in

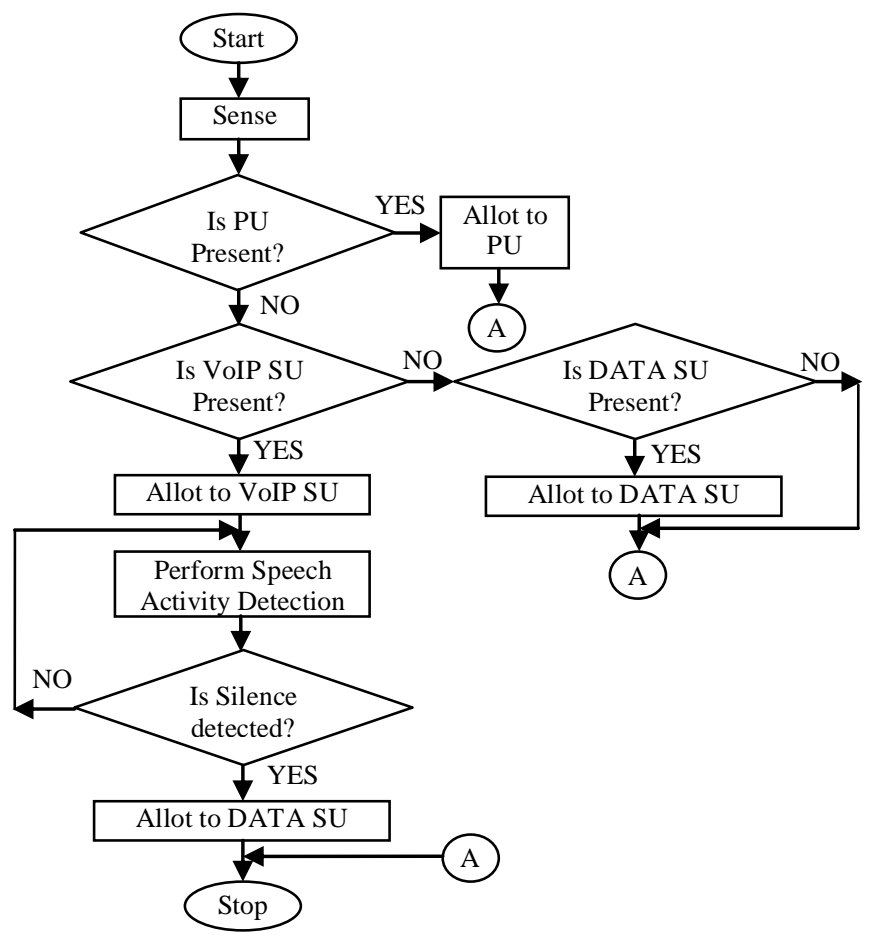

Fig. 1. Flowchart depicting the principle of 2-tier CRN

\section{DESIGN OF MARKOV MODEL For 2-TIER CRN}

This section deals with the design of Markov Models to study the interaction among PU, SUtier1 and SUtier2 under diverse channel conditions. Network is modelled as collection of states where each state denotes channel status with respect to PU, SUtier1 and SUtier2. Let the steady state probability for every such state be denoted by $\mathrm{P}(\mathrm{i}, \mathrm{j}, \mathrm{k}, \mathrm{l}, \mathrm{m})$ where

- $\mathrm{i}=$ total number of active PUs transmitting in CRN,

- $\mathrm{j}=$ total number of active SUtier1 in CRN, that has arrived in the CRN,

- $\mathrm{k}=$ total number of active SUtier2 in CRN, that has been accepted by SUtier1,
- $1=$ current "status" of SUtier1, and

- $\mathrm{m}=$ current "status" of SUtier2

The term "status" denotes the action taken by SU under different network conditions. The various status symbols along with their meanings are described in Table I.

Development of Markov model for 2-tier CRN is carried out incrementally in three phases. Initially, the first tier of CRN is modeled considering appropriate traffic distributions of PU and SUtier1. Secondly, SUtier2 is incorporated into the designed model following the principle of 2-tier CRN as discussed in Section II. Finally, spectrum handoff is incorporated for all SUs in the CRN.

\section{A. Markov Model Design for first tier of CRN with Spectrum Handoff}

Initially, the first tier of CRN is designed using Markov Model. It is obvious that in the absence of any further tier of SUs in the network, the first tier of CRN corresponds to the basic CRN comprising of PUs and a single set of SUs. It is considered that PU and SUtier1 arrive in CRN following Poisson distribution with mean rates $\lambda_{p}$ and $\lambda_{s}$ respectively and have negative exponential service time distribution with mean rates $1 / \mu_{p}$ and $1 / \mu_{s}$ respectively. In order to design the Markov Model, $\mathrm{P}(\mathrm{i}, \mathrm{j}, \mathrm{k}, \mathrm{l}, \mathrm{m})$ is calculated for every possible state. As SUtier2 is not present, $k=0$ for all $\mathrm{P}(\bullet)$ in this scenario.

Spectrum handoff is implemented for SUtier1 such that on arrival of PU in current channel, SUtier1 shifts to the nearest available idle channel. It is to be noted that the implementation of spectrum handoff is dependent on several factors that include underlying MAC protocol, CRN architecture, handoff policies, etc. and hence, its discussion is beyond the scope of this paper. The generalized Markov model for CRN comprising of $N$ channels is developed in Fig. 2 followed by the balance equations guiding the transmission of SUtier1.

TABLE I. STATUS SYMBOLS USED IN MARKOV MODEL

\begin{tabular}{|l|l|l|}
\hline Status Value & Meaning & Definition \\
\hline 0 & $\begin{array}{l}\text { Transmission } \\
\text { Mode }\end{array}$ & $\begin{array}{l}\text { The SU has obtained access to a } \\
\text { channel and is successfully } \\
\text { transmitting. }\end{array}$ \\
\cline { 2 - 3 } 1 & Null Mode & $\begin{array}{l}\text { SU is not performing any } \\
\text { transmission, handoff, blocking or } \\
\text { dropping functions. }\end{array}$ \\
\hline \multirow{2}{*}{2} & $\begin{array}{l}\text { On PU arrival, the SU is } \\
\text { performing spectrum handoff } \\
\text { considering that an idle channel is } \\
\text { available in the system. SU } \\
\text { transmission is suspended } \\
\text { temporarily during the handoff } \\
\text { process. }\end{array}$ \\
\hline Dropping Mode & $\begin{array}{l}\text { SU transmission is suspended } \\
\text { permanently as PU has arrived in } \\
\text { the current channel and there are } \\
\text { no idle channels available in } \\
\text { CRN. }\end{array}$ \\
\hline Blocking Mode & $\begin{array}{l}\text { The incoming SU is not allowed } \\
\text { to gain access to any channel for } \\
\text { initiating transmission as there is } \\
\text { no idle channel left in CRN. }\end{array}$ \\
\hline
\end{tabular}




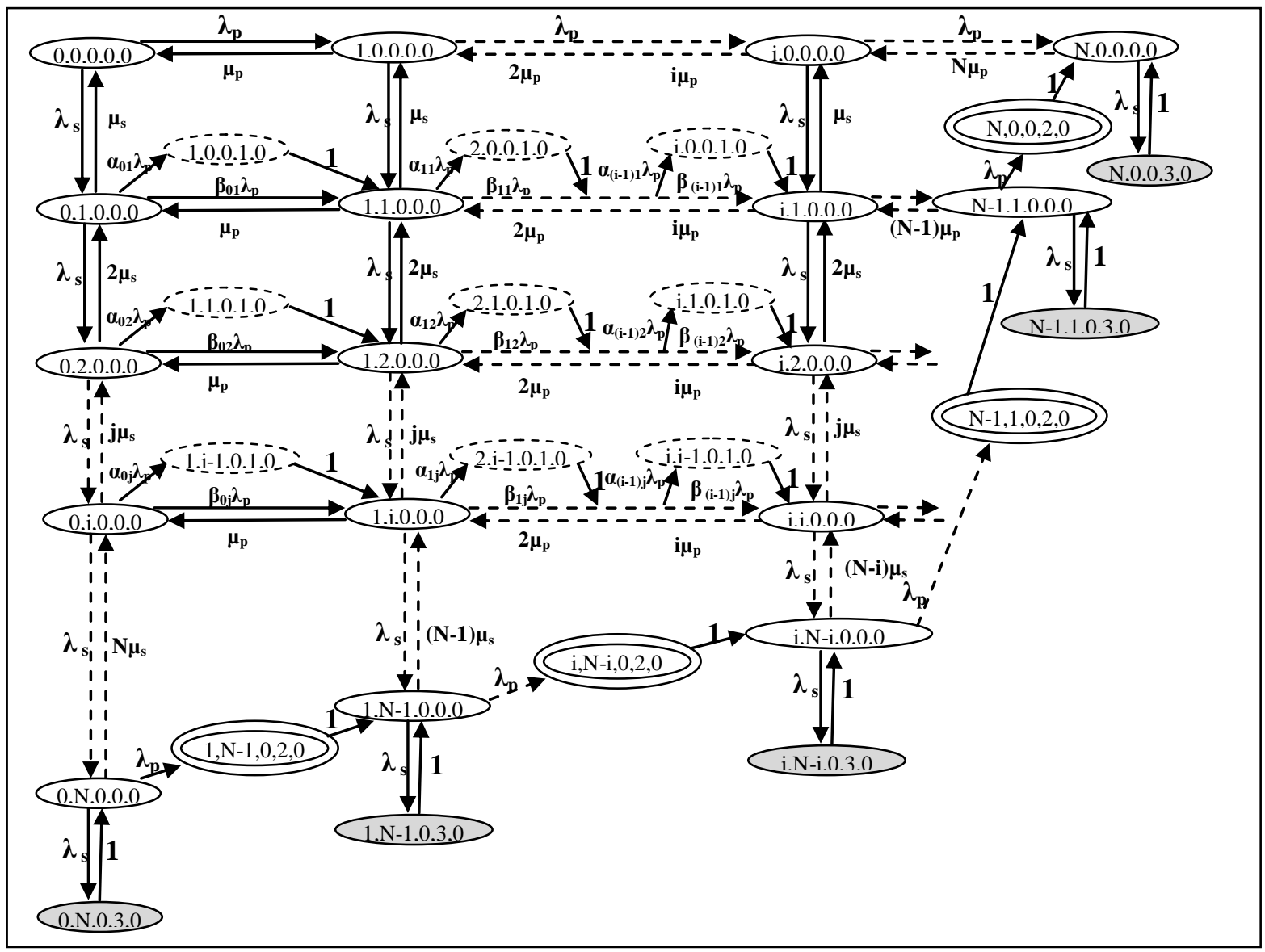

Fig. 2. Markov Model for the first tier of CRN

The balance equations governing the transmission of SUtier1 in the Markov Model for CRN are defined as follows.

i)

ii) (1)

$$
i+j \leq N-1, i=0:\left(\lambda_{p}+\lambda_{s}+j \mu_{s}\right) P(i, j, k, l, m)=\lambda_{s} P(i, j-1, k, l, m)+\mu_{p} P(i+1, j, k, l, m)+(j+1) \mu_{s} P(i, j+1, k, l, m)
$$

$$
\begin{aligned}
& i+j \leq N-1, i \neq 0:\left(\lambda_{p}+\lambda_{s}+j \mu_{s}+i \mu_{p}\right) P(i, j, k, l, m)=\left\{\frac{N-(i-1)-j}{N-(i-1)}\right\} \lambda_{p} P(i-1, j, k, l, m)+\lambda_{s} P(i, j-1, k, l, m)+P(i, j-1, k, 1,0) \\
& +(i+1) \mu_{p} P(i+1, j, k, l, m)+(j+1) \mu_{s} P(i, j+1, k, l, m)
\end{aligned}
$$

iii)

$$
i+j=N, i=0:\left(\lambda_{p}+\lambda_{s}+j \mu_{s}\right) P(i, j, k, l, m)=P(i, j, k, 3,0)+\lambda_{s} P(i, j-1, k, 0,0)
$$

iv)

$$
\begin{aligned}
& i+j=N, i \neq 0::\left(\lambda_{p}+\lambda_{s}+j \mu_{s}+i \mu_{p}\right) P(i, j, k, l, m)=\left\{\frac{N-(i-1)-j}{N-(i-1)}\right\} \lambda_{p} P(i-1, j, k, l, m)+\lambda_{s} P(i, j-1, k, l, m)+P(i, j-1, k, 1,0) \\
& +P(i, j, k, 2,0)+P(i, j, k, 3,0)
\end{aligned}
$$

\section{B. Markov Model Design for 2-Tier CRN with Spectrum Handoff only for SUtierl}

In a 2-tier CRN, each SUtier1 allows SUtier2 to transmit during the silence periods as depicted in Fig. 1. Let SUtier2 arrive in CRN following Poisson distribution with $\lambda_{t}$ as the mean rate and has negative exponential service time distribution with mean rate of $1 / \mu_{t}$. Considering total number of PU and SUtier1 in the network at a certain time interval to be $i$ and $j$ respectively, the maximum number of SUtier2 admitted in CRN is $j$. The addition of SUtier2 by SUtier1 is depicted by a segment of the Markov Model in Fig. 3.

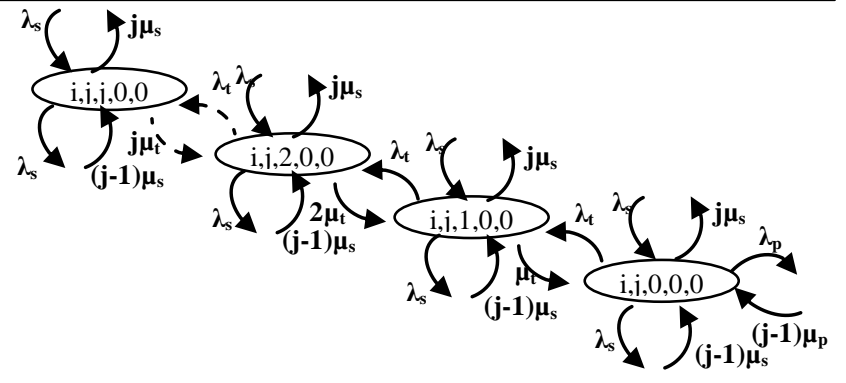

Fig. 3. Admission of SUtier2 in 2-tier CRN 
The maximum system capacity in terms of users admitted in 2-tier CRN is given by,

$$
C p_{\max }=P U+\text { SUtier } 1+\text { SUtier } 2=i+j+j=i+2 j
$$

SUtier2 does not perform spectrum handoff in this model. Rather, it is dropped under three conditions namely, i) at a time when SUtier1 is dropped, ii) when SUtier1 performs spectrum handoff, and iii) after SUtier1 finishes transmission. Therefore, status of SUtier2, as denoted by $m$ in P(i,j,k,1,m), accepts values of 0,2 and 3 depending on its transmission, dropping and blocking mode respectively. At any point of time, status combinations for SUtier 1 and SUtier2 as represented by $\{1, \mathrm{~m}\}$ follow the conditions described in Table II.

Accordingly, the Markov Model for 2-tier CRN (where spectrum handoff is performed by SUtier 1 only) is illustrated in Fig. 4 along with the balance equations for SUtier 1 and SUtier2.
TABLE II. STATUS CONDITIONS For THE DESIGNED MAR KOV MOdEL OF 2-TIER CRN

\begin{tabular}{|c|c|}
\hline Condition & $\underline{\mathbf{n}}$ \\
\hline$m=\{0,2\} \forall m \in\{l, m\}: l=1$ & \multirow{2}{*}{$\begin{array}{l}\text { SUtier2 is dropped when the } \\
\text { corresponding SUtier1 } \\
\text { implements spectrum handoff. }\end{array}$} \\
\hline$l=1 \forall l \in\{l, m\}: m=1$ & \\
\hline$m=\{0,3\} \forall m \in\{l, m\}: l=3$ & \multirow{2}{*}{$\begin{array}{l}\text { The fact that SUtier2 is blocked } \\
\text { from accessing the channel } \\
\text { implies that SUtier1 is already } \\
\text { blocked. }\end{array}$} \\
\hline$l=3 \forall l \in\{l, m\}: m=3$ & \\
\hline$m=\{0,2\} \forall m \in\{l, m\}: l=2$ & \multirow[b]{2}{*}{$\begin{array}{l}\text { Both SUtier1 and SUtier2 } \\
\text { transmissions can be dropped on } \\
\text { the arrival of PU. A special case } \\
\text { occurs when SUtier } 2 \text { transmission } \\
\text { is dropped when the transmission } \\
\text { time interval for SUtier1 is over } \\
\text { and the channel is released. }\end{array}$} \\
\hline $\begin{array}{l}l=0 \forall m \in\{l, m\}: m=2 \text { when } \\
j=k \\
=2 \forall m \in\{l, m\}: m=2 \text { when } \\
j \neq k\end{array}$ & \\
\hline
\end{tabular}

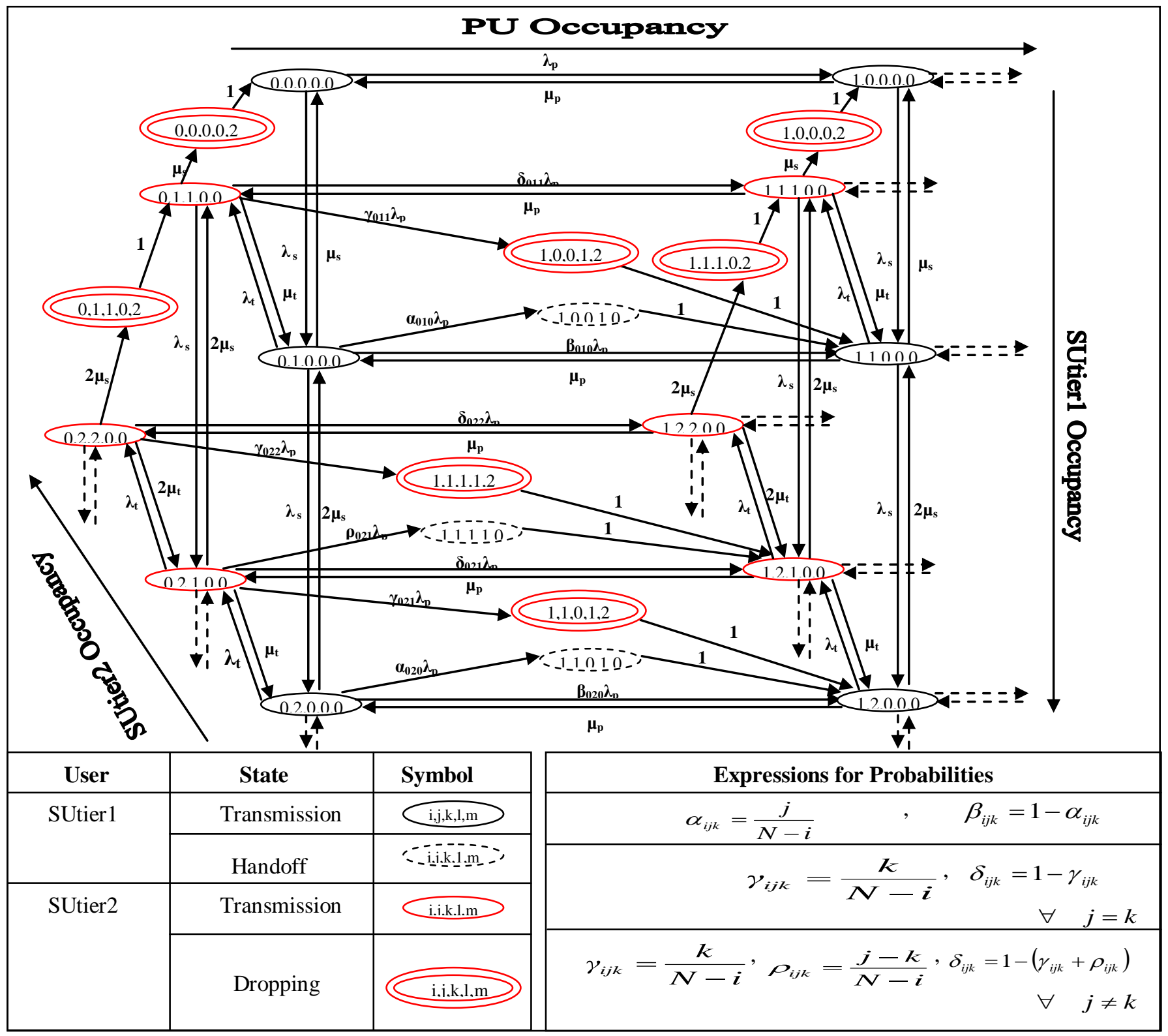

Fig. 4. Markov Model for 2-tier CRN with spectrum handoff only for SUtier1 
The balance equations guiding the transmission of SUtier1 and SUtier2 in Markov Model for 2-tier CRN as per Fig. 4 are defined as follows.

\section{CASE I: SUtier1}

i) $\quad i+j \leq N-1, i=0:\left(\lambda_{p}+\lambda_{s}+\lambda_{t}+j \mu_{s}\right) P(i, j, k, l, m)=\lambda_{s} P(i, j-1, k, l, m)+\mu_{p} P(i+1, j, k, l, m)+(j+1) \mu_{s} P(i, j+1, k, l, m)+\mu_{t} P(i, j, k+1, l, m)(6)$

ii) $i+j \leq N-1, i \neq 0:\left(\lambda_{p}+\lambda_{s}+\lambda_{t}+j \mu_{s}+i \mu_{p}\right) P(i, j, k, l, m)=\beta_{(i-1) j k} \lambda_{p} P(i-1, j, k, l, m)+P(i, j-1, k, 1,0)+\mu_{t} P(i, j, k+1, l, m)$ $+\lambda_{s} P(i, j-1, k, l, m)+(i+1) \mu_{p} P(i+1, j, k, l, m)+(j+1) \mu_{s} P(i, j+1, k, l, m)+P(i, j-1, k, 1,2)$

iii) $i+j=N, i=0:\left(\lambda_{p}+\lambda_{s}+\lambda_{t}+j \mu_{s}\right) P(i, j, k, l, m)=P(i, j, k, 3,0)+\lambda_{s} P(i, j-1, k, l, m)+\mu_{t} P(i, j, k+1, l, m)$

iv) $i+j=N, i \neq 0:\left(\lambda_{p}+\lambda_{s}+\lambda_{t}+j \mu_{s}+i \mu_{p}\right) P(i, j, k, l, m)=\beta_{(i-1) j k} \lambda_{p} P(i-1, j, k, l, m)+\lambda_{s} P(i, j-1, k, l, m)+P(i, j-1, k, 1,0)$ $+\mu_{t} P(i, j, k+1, l, m)+P(i, j, k, 2,0)+P(i, j, k, 3,3)+P(i, j-1, k, 1,2)$

CASE II: SUtier2

$\begin{array}{ll}\text { v) } & i+j \leq N-1, k<j:\left(\lambda_{p}+\lambda_{s}+\lambda_{t}+j \mu_{s}+k \mu_{t}\right) P(i, j, k, l, m)=(k+1) \mu_{t} P(i, j, k+1, l, m)+\lambda_{s} P(i, j-1, k, l, m)+\mu_{p} P(i+1, j, k, l, m) \\ & +\lambda_{t} P(i, j, k-1, l, m)+(j+1) \mu_{s} P(i, j+1, k, l, m)\end{array}$

vi) $i+j \leq N-1, k=j, i=0:\left(\lambda_{p}+\lambda_{s}+j \mu_{s}+k \mu_{t}\right) P(i, j, k, l, m)=(j+1) \mu_{s} P(i, j+1, k, l, m)+\mu_{p} P(i+1, j, k, l, m)+\lambda_{t} P(i, j, k-1, l, m)+P(i, j, k, 0,2)(11)$

vii) $i+j \leq N-1, k=j, i \neq 0:\left(\lambda_{p}+\lambda_{s}+i \mu_{p}+j \mu_{s}+k \mu_{t}\right) P(i, j, k, l, m)=\delta_{(i-1) j k} P(i-1, j, k, l, m)+(i+1) \mu_{p} P(i+1, j, k, l, m)+\lambda_{t} P(i, j, k-1, l, m)$ $+(j+1) \mu_{s} P(i, j+1, k, l, m)+P(i, j, k, 0,2)$

viii) $i+j=N, k<j, i \neq 0:\left(\lambda_{p}+\lambda_{s}+\lambda_{t}+i \mu_{p}+j \mu_{s}+k \mu_{t}\right) P(i, j, k, l, m)=P(i, j-1, k, 1,0)+P(i, j-1, k-1,1,2)+\delta_{(i-1) j k} \lambda_{p} P(i-1, j, k, l, m)$ $+P(i, j, k, 2,2)+P(i, j, k, 2,0)+\lambda_{s} P(i, j-1, k, 0,0)+\lambda_{t} P(i, j, k-1,0,0)+(k+1) \mu_{t} P(i, j, k+1,0,0)+P(i, j, k, 3,0)$

ix) $i+j=N, k=j, i \neq 0:\left(\lambda_{p}+\lambda_{s}+i \mu_{p}+j \mu_{s}+k \mu_{t}\right) P(i, j, k, l, m)=\delta_{(i-1) j k} \lambda_{p} P(i-1, j, k, l, m)+P(i, j, k, 2,2)+P(i, j, k, 2,0)$ $+\lambda_{t} P(i, j, k-1, l, m)+P(i, j, k, 3,3)$

х) $i+j=N, k=j, i=0:\left(\lambda_{p}+\lambda_{s}+j \mu_{s}+k \mu_{t}\right) P(i, j, k, l, m)=P(i, j, k, 3,3)+\lambda_{t} P(i, j, k-1, l, m)$

xi) $i+j=N, k<j, i=0:\left(\lambda_{p}+\lambda_{s}+\lambda_{t}+j \mu_{s}+k \mu_{t}\right) P(i, j, k, l, m)=\lambda_{s} P(i, j-1, k, l, m)+\lambda_{t} P(i, j, k-1, l, m)+(k+1) \mu_{t} P((i, j, k+1, l, m)+P(i, j, k, 3,0)$

C. Design of Markov Model for 2-Tier CRN with Spectrum Handoff for SUtier1 and SUtier2

In this section, Markov Model is designed for 2-tier CRN where both SUtier1 and SUtier2 perform spectrum handoff on sudden PU arrival. As PU arrives in the current channel and SUtier1 shifts to another channel, it sends information about the new channel to SUtier2. Thereafter, SUtier2 reorients its transceiver to frequency band corresponding to new channel and, thus, implements spectrum handoff. However, it must be noted that since admission of SUtier2 in CRN is completely governed by SUtier1, spectrum handoff can be performed by SUtier2 only when corresponding SUtier1 executes spectrum handoff and is represented by the following condition.

$$
m=\{0,1\} \forall m \in\{l, m\}: l=1
$$

where $l, m$ denote the status symbols in $\mathrm{P}(\bullet)$.

Enabling handoff for all SUs in the network implies that as long as there are idle channels available in the system, the average system capacity is close to the maximum system capacity that is expressed in (5). Table III illustrates the conditions under which spectrum handoff can be performed by either only SUtier1 or both SUtier1 and SUtier2.
However, handoff mechanisms fail when all the idle channels are occupied by PUs and SUs. Mathematically, it is represented by, $N=i+j$

where $N, i, j$ denote total number of channels, PU and SUtier1 in CRN respectively.

In this scenario, it can be ascertained from Table III that,

$$
\delta=1-(\gamma+\rho)=N-(i+j)=0
$$

Any further arrival of PU results in two cases.

- Case 1: $k<j, N>(i+j)$

Only SUtier1 is dropped as there is no SUtier2 in this channel. The probability of SUtier1 being dropped on PU arrival is given by,

$$
\gamma=\frac{j}{N-i}
$$

- Case 2: $k=j, N>(i+j)$

Both SUtier1 and SUtier2 are dropped on arrival of PU with probability as expressed in (21). 
- Case 3: $k=j, N=(i+j)$

$$
\rho=\frac{j-k}{N-i}
$$

SUtier1 and SUtier2 are dropped as PU arrives with probability $=1$.

The complete Markov Model for 2-tier CRN with spectrum handoff implemented by all SUs is depicted in Fig. 5. Symbols as used in Fig. 4 are applied to denote the states in Fig. 5. The most significant balance equations for SUtier1 and SUtier2 corresponding to Fig. 5 are given as under.

The balance equations governing transmission of SUtier1 and SUtier2 in 2-tier CRN as per Fig. 5 are defined as follows.

CASE I: SUtier1

i) $\quad i+j \leq N-1, i=0:\left(\lambda_{p}+\lambda_{s}+\lambda_{t}+j \mu_{s}\right) P(i, j, k, l, m)=\lambda_{s} P(i, j-1, k, l, m)+\mu_{p} P(i+1, j, k, l, m)+(j+1) \mu_{s} P(i, j+1, k, l, m)+\mu_{t} P(i, j, k+1, l, m)(22)$

ii) $i+j \leq N-1, i \neq 0:\left(\lambda_{p}+\lambda_{s}+\lambda_{t}+j \mu_{s}+i \mu_{p}\right) P(i, j, k, l, m)=\beta_{(i-1) j k} \lambda_{p} P(i-1, j, k, l, m)+P(i, j-1, k, 1,0)+\mu_{t} P(i, j, k+1, l, m)$

$+\lambda_{s} P(i, j-1, k, l, m)+(i+1) \mu_{p} P(i+1, j, k, l, m)+(j+1) \mu_{s} P(i, j+1, k, l, m)+P(i, j-1, k, 1,2)$

iii) $i+j=N, i=0:\left(\lambda_{p}+\lambda_{s}+\lambda_{t}+j \mu_{s}\right) P(i, j, k, l, m)=P(i, j, k, 3,0)+\lambda_{s} P(i, j-1, k, l, m)+\mu_{t} P(i, j, k+1, l, m)$

iv) $i+j=N, i \neq 0:\left(\lambda_{p}+\lambda_{s}+\lambda_{t}+j \mu_{s}+i \mu_{p}\right) P(i, j, k, l, m)=\beta_{(i-1) j k} \lambda_{p} P(i-1, j, k, l, m)+\lambda_{s} P(i, j-1, k, l, m)+P(i, j-1, k, 1,0)$

$+\mu_{t} P(i, j, k+1, l, m)+P(i, j, k, 2,0)+P(i, j, k, 3,3)+P(i, j-1, k, 1,2)$

CASE II: SUtier2

v) $i+j \leq N-1, k<j:\left(\lambda_{p}+\lambda_{s}+\lambda_{t}+j \mu_{s}+k \mu_{t}\right) P(i, j, k, l, m)=(k+1) \mu_{t} P(i, j, k+1, l, m)+\lambda_{s} P(i, j-1, k, l, m)+\mu_{p} P(i+1, j, k, l, m)$

$+\lambda_{t} P(i, j, k-1, l, m)+(j+1) \mu_{s} P(i, j+1, k, l, m)$

vi) $i+j \leq N-1, k=j, i=0:\left(\lambda_{p}+\lambda_{s}+j \mu_{s}+k \mu_{t}\right) P(i, j, k, l, m)=(j+1) \mu_{s} P(i, j+1, k, l, m)+\mu_{p} P(i+1, j, k, l, m)+\lambda_{t} P(i, j, k-1, l, m)+P(i, j, k, 0,2)$

vii) $i+j \leq N-1, k=j, i \neq 0:\left(\lambda_{p}+\lambda_{s}+i \mu_{p}+j \mu_{s}+k \mu_{t}\right) P(i, j, k, l, m)=\delta_{(i-1) j k} P(i-1, j, k, l, m)+(i+1) \mu_{p} P(i+1, j, k, l, m)+\lambda_{t} P(i, j, k-1, l, m)$

$+(j+1) \mu_{s} P(i, j+1, k, l, m)+P(i, j, k, 0,2)+P(i, j-1, k-1,1,1)$

viii) $i+j=N, k<j, i \neq 0:\left(\lambda_{p}+\lambda_{s}+\lambda_{t}+i \mu_{p}+j \mu_{s}+k \mu_{t}\right) P(i, j, k, l, m)=P(i, j-1, k, 1,0)+P(i, j-1, k-1,1,1)+\delta_{(i-1) j k} \lambda_{p} P(i-1, j, k, l, m)$

$+P(i, j, k, 2,2)+P(i, j, k, 2,0)+\lambda_{s} P(i, j-1, k, 0,0)+\lambda_{t} P(i, j, k-1,0,0)+(k+1) \mu_{t} P(i, j, k+1,0,0)+P(i, j, k, 3,0)$

ix) $i+j=N, k=j, i \neq 0:\left(\lambda_{p}+\lambda_{s}+i \mu_{p}+j \mu_{s}+k \mu_{t}\right) P(i, j, k, l, m)=\delta_{(i-1) j k} \lambda_{p} P(i-1, j, k, l, m)+P(i, j, k, 2,2)+P(i, j, k, 2,0)$

$+\lambda_{t} P(i, j, k-1, l, m)+P(i, j, k, 3,3)$

х) $i+j=N, k=j, i=0:\left(\lambda_{p}+\lambda_{s}+j \mu_{s}+k \mu_{t}\right) P(i, j, k, l, m)=P(i, j, k, 3,3)+\lambda_{t} P(i, j, k-1, l, m)$

xi)

$i+j=N, k<j, i=0:\left(\lambda_{p}+\lambda_{s}+\lambda_{t}+j \mu_{s}+k \mu_{t}\right) P(i, j, k, l, m)=\lambda_{s} P(i, j-1, k, l, m)+\lambda_{t} P(i, j, k-1, l, m)+(k+1) \mu_{t} P(i, j, k+1, l, m)+P(i, j, k, 3,0)$

TABLE III. DIFFERENT HANDOFF AND DROPPING CONDITIONS For SUTIER1 AND SUTIER2

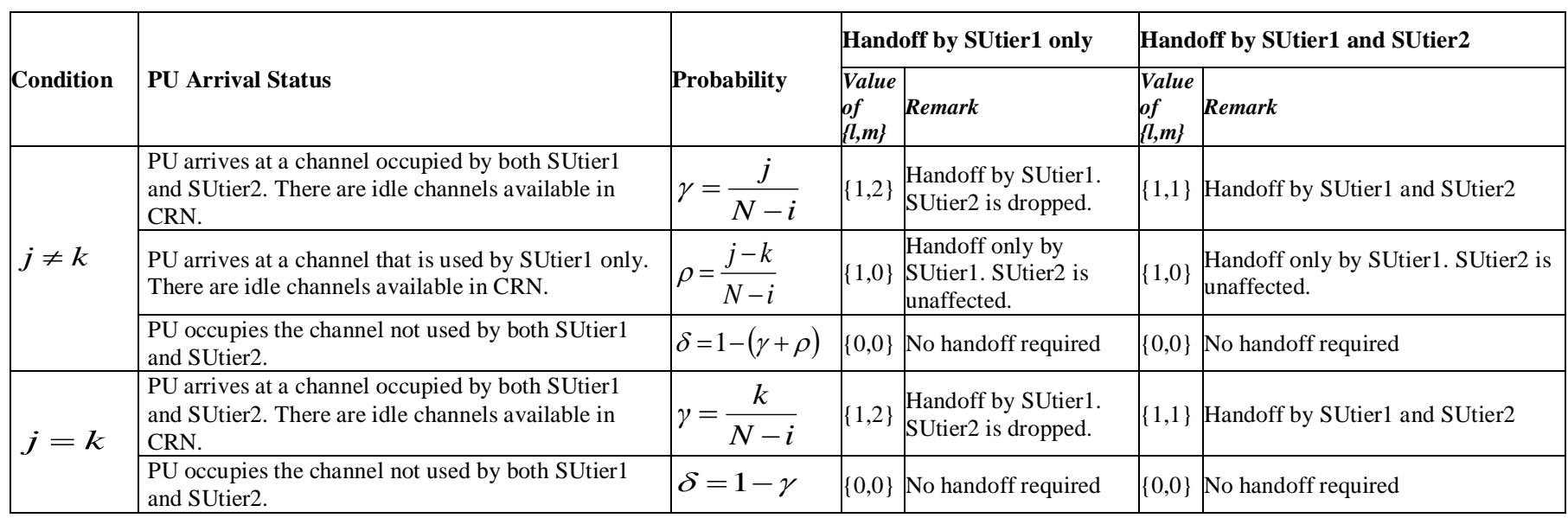




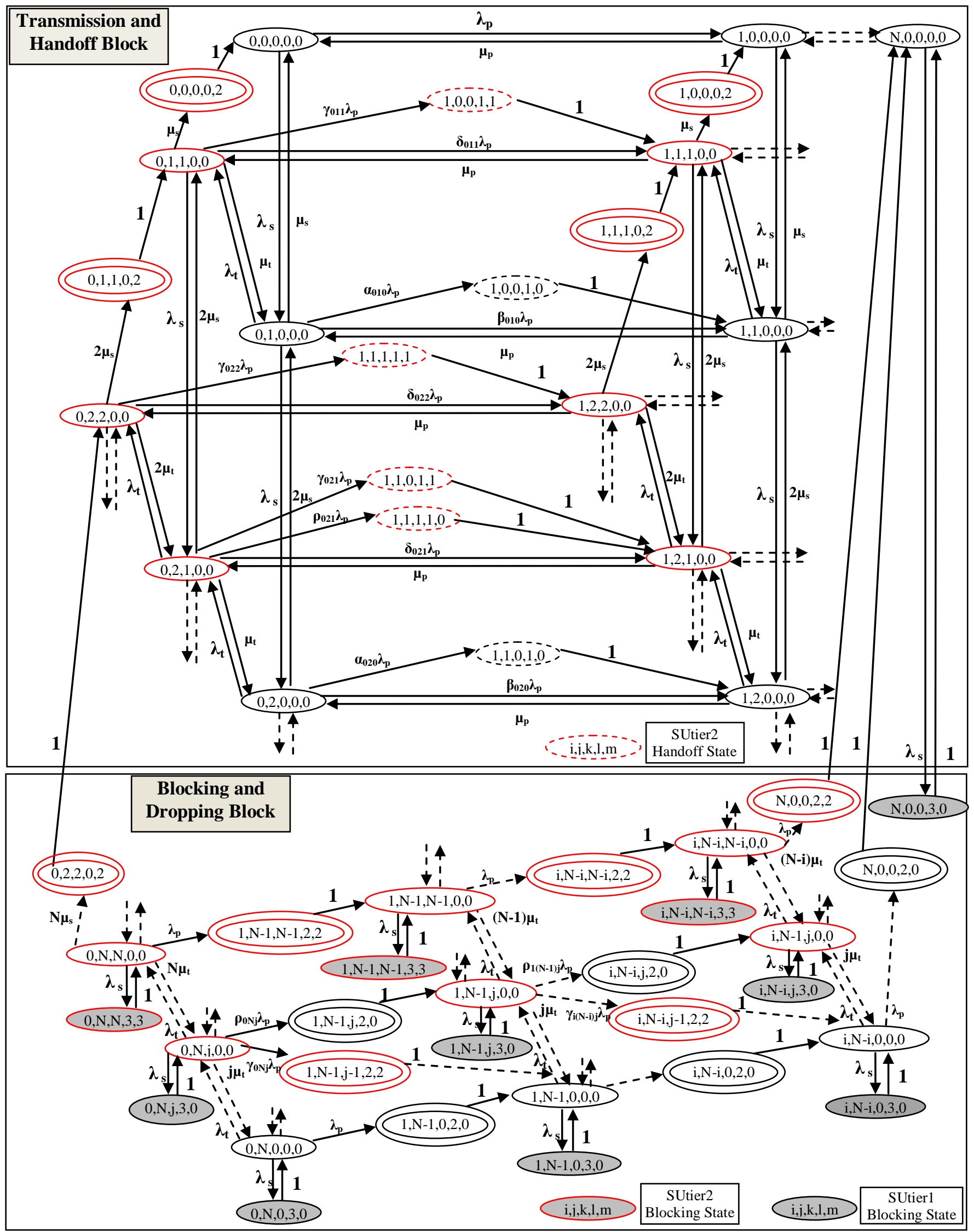

Fig. 5. Complete Markov Model for 2-tier CRN with spectrum handoff for both SUtier1 and SUtier2 


\section{MATHEMATICAL FORMULATION OF SU ThroUghPUT IN 2-TIER CRN}

A mathematical expression is derived to obtain the throughput for the $2^{\text {nd }}$ SUtier 1 that arrives in the proposed 2tier spectrum handoff enabled CRN. It must be noted that the number of available idle channels varies significantly depending on total number of spectrum handoff instances performed by existing SUs. Similarly, the number of SUs to get access to idle channels depends on the total number of SUtier2 supported by SUtier1. Let $m(t)$ be the overall number of available idle channels in CRN. Considering the effects of imperfect sensing by SUtier1 (false alarm and miss-detection), the total number of measured unoccupied channels as expressed in [17] is modified with respect to a particular time interval $t$ and is defined as,

$m^{\prime}(t)=m(t)-m(t) \times p_{f}(\cdot)+(M-m(t)) \times\left(1-p_{d}(\cdot)\right)$

Let $P h_{b}(a)$ be the probability of spectrum handoff performed by $b$ th SU in tier 1 to shift from the current channel $a^{\prime}$ to $a^{\text {th }}$ channel. Therefore, the throughput for the $b^{\text {th }}$ SUtier1 having transmission rate $R_{a},(V o I P)$ in a particular idle channel $a^{\prime}$ at a time interval $t$ is given by,

$C_{b}^{\text {SUtier1 }}(t)=\left(1-P h_{b}(a)\right)\left\{R_{a^{\prime}}(V o I P)\left(\begin{array}{c}m^{\prime}(t)-1 \\ 1\end{array}\right)\left(\frac{1}{m^{\prime}(t)-1}\right)\left(1-\frac{1}{m^{\prime}(t)-1}\right)^{j-1}\right\}$

where $j=$ total number of SUs in the system

Let $P_{s s}(\bullet)$ be a binary variable that defines whether SUtier2 is granted access by SUtier 1 and is defined as follows.

$$
\begin{aligned}
P_{s s}(b) & =1 \text { when } b^{\text {th }} \text { SUtier } 1 \text { allows SUtier } 2 \text { to transmit } \\
& =0 \text { otherwise. }
\end{aligned}
$$

Accordingly, throughput of SUtier2 with transmission rate as $R_{a}(D A T A)$ in the $a^{\text {th }}$ channel at time interval $t$ is given by,

$$
C_{b}^{\text {SUtier } 2}(t)=P_{s s}(b)\left\{R_{a^{\prime}}(D A T A)\left(\begin{array}{c}
m^{\prime}(t)-1 \\
1
\end{array}\right)\left(\frac{1}{m^{\prime}(t)-1}\right)\left(1-\frac{1}{m^{\prime}(t)-1}\right)^{j-2}\right\}
$$

Combining (34) and (36), the total throughput for a particular set of SUtier1 and SUtier2 is expressed in (39).

There are several possibilities with respect to allotment of an idle channel to SUtier1 and is depicted as a three-layered tree in Fig. 6 . For the $2^{\text {nd }}$ SUtier1 in the system, the first layer determines whether the preceding SUtier1 grants access to SUtier2 or not. The second layer specifies the probabilities with which the $1^{\text {st }}$ SUtier1 performs spectrum handoff in different channels. The third layer indicates the different spectrum handoff probabilities for the $2^{\text {nd }}$ SUtier 1 .

A special case occurs when the $1^{\text {st }}$ SUtier1 performs repeated handoff and finally occupies the penultimate channel. In this condition, the $2^{\text {nd }}$ SUtier1 occupies only the last available idle channel and is dropped on the event of any further PU arrival as it cannot perform any spectrum handoff. Let $C_{2 n d S U t h r o u}$ denote the throughput of $2^{\text {nd }}$ SUtier1 corresponding to the second layer of the tree. The general expression for $C_{2 n d S U t h r o u}$ is derived in (36). It is further modified to include the different conditions of spectrum handoff as per Fig. 6 and is expressed in (40).

Let $C_{2 n d S U}$ be the overall throughput of $2^{\text {nd }}$ SUtier 1 at the topmost layer of the tree and is given by,

$$
\begin{gathered}
C_{2 n d S U}(t)=P_{s s}(1) C_{2 n d S U \text { throu }}(t)+\left(1-P_{s s}(1)\right) C_{2 n d S U \text { throu }}(t) \\
\text { V. PERFORMANCE ANALYSIS }
\end{gathered}
$$

This section analyzes the developed Markov models to establish the superiority of 2-tier CRN over basic CRN and also records significant performance improvement after incorporating spectrum handoff in 2-tier CRN. The key parameters that are used to analyze the performance improvement of 2-tier CRN over basic CRN include SU transmission, spectrum handoff, blocking and dropping probabilities and overall SU throughput.

Let $P_{L}$ denote limiting probability of SU acceptance by available idle channel in CRN and is expressed as follows.

$$
P_{L}=\sum_{\substack{i=0, j=N-i}}^{N-1} P(i, j, 0,0,0)
$$

$C_{\text {SUthro }}(t)=\left(1-P h_{b}(a)\right)\left[\left\{R_{a^{\prime}}(V o I P)\left(\begin{array}{c}m^{\prime}(t)-1 \\ 1\end{array}\right)\left(\frac{1}{m^{\prime}(t)-1}\right)\left(1-\frac{1}{m^{\prime}(t)-1}\right)^{j-1}\right\}+P_{s s}(b)\left\{R_{a^{\prime}}(D A T A)\left(\begin{array}{c}m^{\prime}(t)-1 \\ 1\end{array}\right)\left(\frac{1}{m^{\prime}(t)-1}\right)\left(1-\frac{1}{m^{\prime}(t)-1}\right)^{j-2}\right\}\right]$

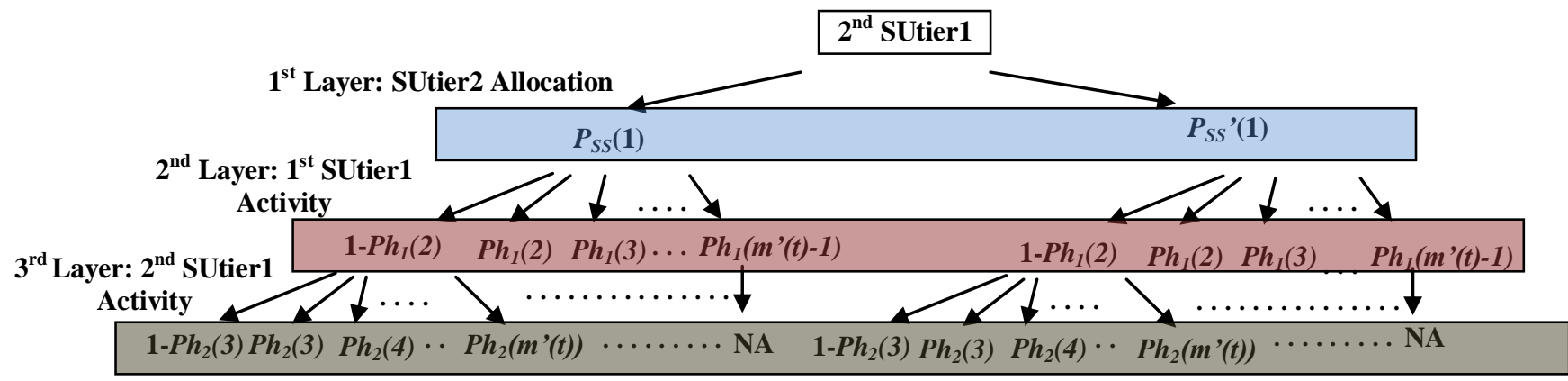

Fig. 6. Schematic Representation of all possibilities regarding channel allocation for $2^{\text {nd }}$ SUtier1 on arrival in CRN 


$$
\begin{aligned}
& C_{2 n d S \text { throu }}(t)=\left[\{ ( 1 - P h _ { 1 } ( 2 ) ) \} \left[\left(1-P h_{2}(3)\right)\left[R_{2}(\operatorname{VoIP})\left(\begin{array}{c}
m^{\prime}(t)-1 \\
1
\end{array}\right)\left(\frac{1}{m^{\prime}(t)-1}\right)\left(1-\frac{1}{m^{\prime}(t)-1}\right)^{l}+P_{s s}(2) R_{2}(D A T A)\left(\begin{array}{c}
m^{\prime}(t)-1 \\
1
\end{array}\right)\left(\frac{1}{m^{\prime}(t)-1}\right)\left(1-\frac{1}{m^{\prime}(t)-1}\right)^{m}\right]\right.\right. \\
& +\left[\sum_{k=3}^{m^{\prime}(t)-1} P h_{2}(k)\left[R_{k}(\operatorname{VoI})\left(\begin{array}{c}
m^{\prime}(t)-k+1 \\
1
\end{array}\right)\left(\frac{1}{m^{\prime}(t)-k+1}\right)\left(1-\frac{1}{m^{\prime}(t)-k+1}\right)^{l}+P_{s s}(2) R_{k}(D A T A)\left(\begin{array}{c}
m^{\prime}(t)-k+1 \\
1
\end{array}\right)\left(\frac{1}{m^{\prime}(t)-k+1}\right)\left(1-\frac{1}{m^{\prime}(t)-k+1}\right)^{m}\right]\right] \\
& +\left[P h_{2}\left(m^{\prime}(t)\right)\left[R_{m^{\prime}(t)}(V o I P)+P S s(2) R_{m^{\prime}(t)}(D A T A)\right]+\left[( P h _ { 1 } ( 2 ) ) \left[\left(1-P h_{2}(4)\right)\left[R_{3}(\operatorname{VoIP})\left(\begin{array}{c}
m^{\prime}(t)-2 \\
1
\end{array}\right)\left(\frac{1}{m^{\prime}(t)-2}\right)\left(1-\frac{1}{m^{\prime}(t)-2}\right)^{l}+P_{s s}(2) R_{3}(D A T A)\left(\begin{array}{c}
m^{\prime}(t)-2 \\
1
\end{array}\right)\left(\frac{1}{m^{\prime}(t)-2}\right)\left(1-\frac{1}{m^{\prime}(t)-2}\right)^{m}\right]\right.\right.\right. \\
& +\left[\sum_{k=4}^{m^{\prime}(t)-1} P h_{2}(k)\left[R_{k}(\operatorname{VoIP})\left(\begin{array}{c}
m^{\prime}(t)-k+1 \\
1
\end{array}\right)\left(\frac{1}{m^{\prime}(t)-k+1}\right)\left(1-\frac{1}{m^{\prime}(t)-k+1}\right)^{l}+P_{s s}(2) R_{k}(D A T A)\left(\begin{array}{c}
m^{\prime}(t)-k+1 \\
1
\end{array}\right)\left(\frac{1}{m^{\prime}(t)-k+1}\right)\left(1-\frac{1}{m^{\prime}(t)-k+1}\right)^{m}\right]\right] \\
& +\left\lfloor P h_{2}\left(m^{\prime}(t)\right)\left|R_{m^{\prime}(t)}(\operatorname{VoIP})+P s s(2) R_{m^{\prime}(t)}(D A T A) \rrbracket+\ldots \ldots . .+P h_{1}\left(m^{\prime}(t)-1\right)\right| R_{m^{\prime}(t)}(\operatorname{VoIP})+\operatorname{Pss}(2) R_{m^{\prime}(t)}(D A T A)\right\rfloor \\
& \text { where } l=j-3, m=j-4 \forall \operatorname{Pss}(1) \text {, } \\
& l=j-2, m=j-3 \forall(1-P s s(1))
\end{aligned}
$$

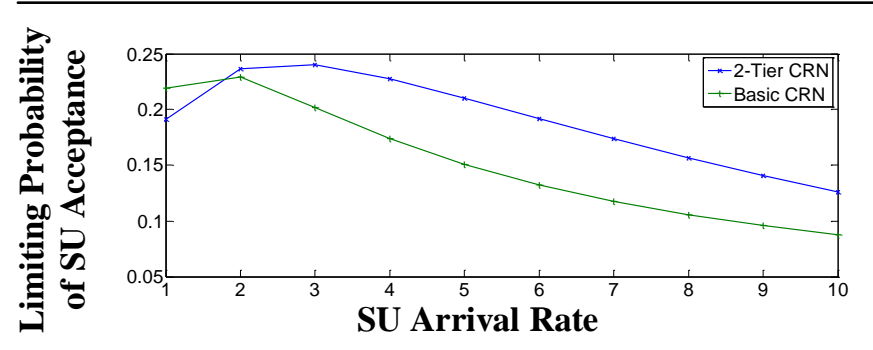

Fig. 7. Variation in limiting probability of SU acceptance by CRN with SU arrival rate

It is observed from Fig. 7 that 2-tier CRN provides higher probability of $\mathrm{SU}$ acceptance than basic $\mathrm{CRN}$ and thus reduces the overall blocking probability (denoted by $P_{B}$ ).

Let $P_{D}$ define the steady state dropping probability that $\mathrm{SU}$ transmission is dropped before scheduled transmission interval is over. The expression for $P_{D}$ is derived from [18] and is expressed in (41) as per the designed Markov Model.

$$
P_{D}=\frac{\sum P_{d r o p}}{\left(1-P_{B}\right) \lambda_{s}}
$$

where $P_{\text {drop }}=\sum P(i, j, k, l, m) \forall(l, m)=(2,2)|(2,0)|(0,2)$

Therefore, spectrum handoff must be performed by SUs to shift to available idle channels on PU arrival to reduce $P_{D}$. Let $P_{\text {handoff_tierl }}$ and $P_{\text {handoff_tier2 }}$ be the probabilities of spectrum handoff performed by SUtier1 and SUtier2 respectively and are expressed as follows.

$$
\begin{gathered}
P_{\text {handoff_tier } 1}(i, j)=\frac{\sum P(i, j, 0,1,0)}{\left(1-P_{B}\right) \lambda_{s}} \\
P_{\text {handoff_tier } 2}(i, j)=\frac{\sum P(i, j, 0,1,1)}{\left(1-P_{B}\right) \lambda_{s}}
\end{gathered}
$$

The dropping and handoff probabilities for SUs in 2-tier CRN are plotted in Fig. 8 and Fig. 9 respectively, for two scenarios that correspond to i) spectrum handoff by SUtier1 only, and ii) spectrum handoff by SUtier1 and SUtier2. It is imperative that when SUtier1 performs spectrum handoff, SUtier2 is either dropped or else it must also perform handoff. This situation is clearly reflected in Fig. 8 where $P_{D}$ is less for scenario 2 compared to scenario1.

Therefore, reduction in blocking and dropping probabilities must increase SU throughput in spectrum handoff enabled 2tier CRN. This is illustrated in Fig. 10 that plots the probability of successful transmission by SUs with increase in PU activity. It is observed from the figure that 2-tier CRN with complete spectrum handoff has the highest probability of transmission

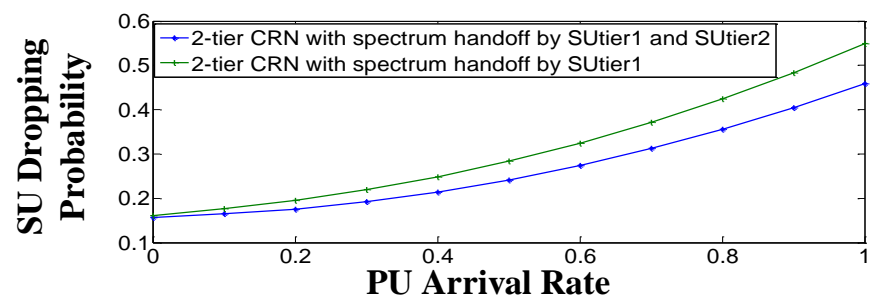

Fig. 8. Variation in SU dropping probability in CRN with PU arrival rate

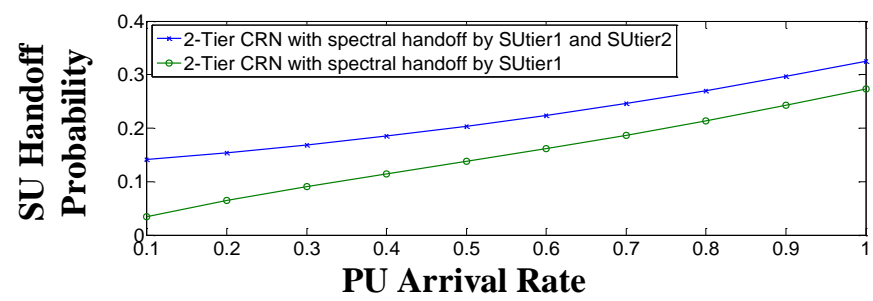

Fig. 9. Performance of 2-tier CRN with respect to SU handoff probability for varying PU arrival rate

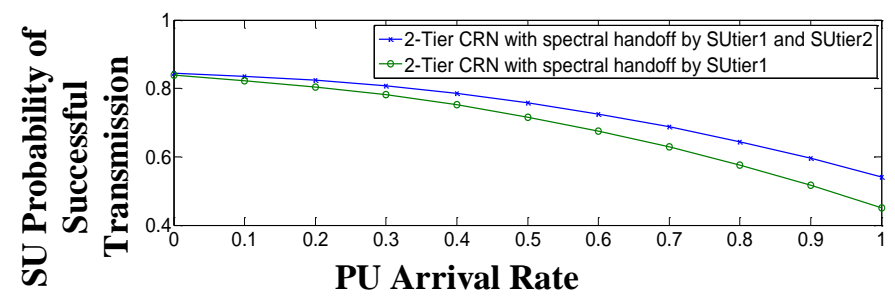

Fig. 10. Variation in probability of successful transmission by SU in CRN with PU arrival rate 


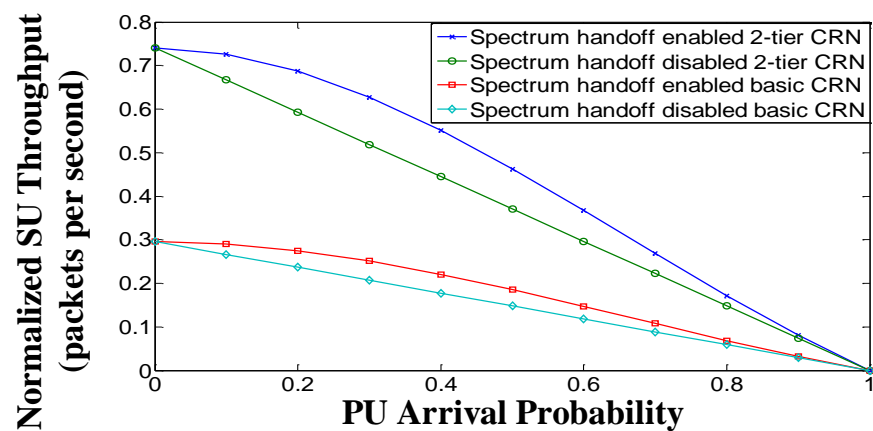

Fig. 11. Effect of spectrum handoff on normalized SU throughput with varying PU arrival probability for basic and 2-tier CRN

The normalized SU throughput as obtained from (37) is plotted in Fig. 11 for increasing probability of PU arrival on the current channel with respect to 2-tier CRN and basic CRN. As observed from the figure, spectrum handoff enabled 2-tier CRN provides the highest $\mathrm{SU}$ throughput compared to the other scenarios. In addition, CRN implementing spectrum handoff performs better as it records almost $25 \%$ enhancement in throughput (for $0.5 \mathrm{PU}$ arrival probability) compared to spectrum handoff disabled CRN. However, as PU activity increases in CRN, number of idle channels reduces drastically. Under such circumstances, throughput for SUs supporting spectrum handoff decreases as reflected in Fig. 11.

Thus, observation from the designed Markov Model in Fig. 10 is validated using output derived from the mathematical model as represented in Fig. 11.

\section{CONCLUSION}

This work has addressed the problem of limited system capacity in basic CRN by designing a 2-tier CRN that allows more number of SUs in the system. While the first tier of SUs involve in VoIP communication, the second tier of SUs exploit the silence periods in VoIP transmission to send data. Markov Models have been designed in this regard to highlight the difference between basic and 2-tier CRN. Spectrum handoff has also been incorporated in the developed Markov Model for performance enhancement. Analysis of the Markov models has recorded significant reduction in SU dropping and blocking probabilities in spectrum handoff enabled 2-tier CRN along with increase in successful transmission probabilities for SUs. A mathematical framework to study SU behavior has been formulated, that has recorded highest SU throughput after enabling spectrum handoff in 2-tier CRN and has, thus, confirmed the inference drawn from the Markov models. The 2-tier CRN is being studied presently to devise appropriate MAC protocols and spectrum handoff policies apart from architectural modifications and channel reservation schemes.

\section{ACKNOWLEDGMENT}

The first author acknowledges the support of INSPIRE Fellowship 2012 from DST, Govt. of India.

\section{REFERENCES}

[1] Federal Communications Commission, "Spectrum policy task force report," ET Docket No.02-135, 2002
[2] U.S Department of Commerce, "United States Frequency Allocations: The Radio Spectrum", 2011.

[3] Federal Communications Commission, "Notice of proposed rule making and order: Facilitating opportunities for flexible, efficient, and reliable spectrum use employing cognitive radio technologies," ET Docket No. 03-108, February 2005.

[4] T. Y'ucek and H. Arslan, "A survey of spectrum sensing algorithms for cognitive radio applications," IEEE Communications Surveys and Tutorials, vol.11, no.1, pp.116-130, 2009, doi: 10.1109/SURV.2009.090109.

[5] J. Mitola III and G. Q. Maguire, Jr, "Cognitive radio: making software radios more personal," IEEE Personal Communications, vol. 6, no. 4, pp. 13-18, August 1999, doi: 10.1109/98.788210.

[6] F. Akyildiz, W. Y. Lee, M. C. Vuran and S. Mohanty, "NeXt generation / dynamic spectrum access / cognitive radio wireless networks: A survey," Computer Networks Journal (Elsevier) 50, pp. 2127- 2159, September 2006.

[7] C. Qian, L. Ying-Chang, M. Motani and W. Wai-Choong, "A TwoLevel MAC Protocol Strategy for Opportunistic Spectrum Access in Cognitive Radio Networks," IEEE Transactions on Vehicular Technology, vol.60, no.5, pp.2164-2180, June 2011, doi: 10.1109/TVT.2011.2141694.

[8] T.T. Le and L.L. Bao, "Distributed MAC Protocol for Cognitive Radio Networks: Design, Analysis, and Optimization," IEEE Transactions on Vehicular Technology, vol. 60, no. 8, pp. 3990-4003, October 2011, doi: 10.1109/TVT.2011.2165325

[9] L. Wang, C. Wang and C. Chang, "Modeling and Analysis for Spectrum Handoffs in Cognitive Radio Networks," IEEE Transactions on Mobile Computing, vol. 11, no. 9, pp. 1499-1513, September 2012, doi: 10.1109/TMC.2011.155.

[10] T. Chakraborty, I.S. Misra, and S.K. Sanyal, "Selection of optimal transmission time in cognitive radio network for efficient VoIP performance", Proc. of Fifth International Conference on Computers and Devices for Communication (CODEC), pp.1-4, India, 17-19 December 2012, doi: 10.1109/CODEC.2012.6509230.

[11] P. Wang, L. Xiao, Shidong Zhou and J. Wang, "Optimization of detection time for channel efficiency in cognitive radio systems," Proc. of Wireless Communications and Networking Conference, (WCNC 2007), pp.111-115, Hong-Kong, March 11-15, 2007.

[12] S. Srinivasa and S. Jafar, "How much spectrum sharing is optimal in cognitive radio networks?," IEEE Transactions on Wireless Communications, vol. 7, no. 10, pp. 4010-4018, October 2008, doi:10.1109/T-WC.2008.070647

[13] B, Khasnabish, Implementing Voice over IP. Wiley-Interscience, John Wiley \& Sons, Inc., 2003.

[14] Z. Xiaorong, S. Lianfeng and T.-S.P.Yum, "Analysis of Cognitive Radio Spectrum Access with Optimal Channel Reservation," IEEE Communications Letters, vol.11, no.4, pp.304-306, April 2007, doi: 10.1109/LCOM.2007.348282.

[15] Y.R. Kondareddy, N. Andrews, and P. Agrawal, "On the capacity of secondary users in a cognitive radio network," Proc. of IEEE Sarnoff Symposium (SARNOFF '09), pp.1-5, U.S.A., March 30 -April 1, 2009.

[16] I.A. Qaimkhani and E. Hossain, "Efficient silence suppression and call admission control through contention-free medium access for VoIP in WiFi networks," IEEE Communications Magazine, vol. 46, no. 1, pp. 90-99, January 2008, doi: 10.1109/MCOM.2008.4427236.

[17] H. Lee and D. Cho, "Capacity Improvement and Analysis of VoIP Service in a Cognitive Radio System," IEEE Transactions on Vehicular Technology, vol. 59, no. 4, pp. 1646-1651, May 2010, doi: 10.1109/TVT.2009.2039503.

[18] J. Martinez-Bauset, V. Pla and D. Pacheco-Paramo, "Comments on "analysis of cognitive radio spectrum access with optimal channel reservation"," IEEE Communications Letters, vol.13, no.10, pp.739, October 2009, doi: 10.1109/LCOMM.2009.090668. 